Copyright @ 2005 IEEE.

Reprinted from the Annual Meeting of the IEEE Lasers and ElectroOptics Society (18th : 2005 : Sydney, Australia):pp.607-608

This material is posted here with permission of the IEEE. Such permission of the IEEE does not in any way imply IEEE endorsement of any of the University of Adelaide's products or services. Internal or personal use of this material is permitted. However, permission to reprint/republish this material for advertising or promotional purposes or for creating new collective works for resale or redistribution must be obtained from the IEEE by writing to pubs-permissions@ieee.org.

By choosing to view this document, you agree to all provisions of the copyright laws protecting it. 


\title{
High Performance Bragg Gratings in Chalcogenide Glass Rib Waveguides Written with a Modified Sagnac Interferometer: Fabrication and Characterization
}

\author{
M. Shokooh-Saremi ${ }^{1}$, V. G. Ta'eed ${ }^{1}$, N. J. Baker ${ }^{1}$, I. C. M. Littler ${ }^{1}$, D. J. Moss ${ }^{1}$, B. J. Eggleton ${ }^{1}$, \\ Y. Ruan ${ }^{2}$ and B. Luther-Davies ${ }^{2}$ \\ ${ }^{I}$ Centre for Ultrahigh-bandwidth Devices for Optical Systems (CUDOS), School of Physics, University of Sydney, \\ Sydney NSW 2006, Australia, E-mail: mehrdad@physics.usyd.edu.au \\ ${ }^{2}$ Centre for Ultrahigh-bandwidth Devices for Optical Systems (CUDOS), Australian National University, \\ Canberra, ACT, Australia
}

\begin{abstract}
We report high performance Bragg gratings in $\mathrm{As}_{2} \mathrm{~S}_{3}$ chalcogenide glass rib waveguides, written with a modified Sagnac interferometer for the first time. Grating growth dynamics obtained from an in-situ monitoring system are presented and analyzed.
\end{abstract}

Chalcogenide glasses have attracted significant attention in the past few years as promising nonlinear optical materials in the infrared telecommunications window. $\mathrm{As}_{2} \mathrm{~S}_{3}$ is one of these glasses that exhibits a high third order nonlinearity ( 400 times of silica), low two photon absorption (and hence good figure of merit), and furthermore its optical properties can be stoichiometrically tuned. In addition, it is photosensitive when exposed to visible light near its optical absorption edge $(\sim 514-632 \mathrm{~nm})$ [1-2]. This phenomenon has been utilized in fabrication of optical devices such as waveguides and gratings [3-6]. To date, however, the performance specifications of Bragg gratings in chalcogenide waveguides have been limited.

In this paper, we present high performance waveguide gratings in $\mathrm{As}_{2} \mathrm{~S}_{3}$ chalcogenide glass rib waveguides. These gratings are of high enough quality to be appropriate for advanced all-optical signal processing applications, such as optical regeneration. The gratings are written using a highly stable holographic writing setup based on a modified Sagnac interferometer. This setup allows easy Bragg wavelength tuning with high quality grating apodization, and has enabled us to achieve very strong, well apodized Bragg gratings in $\mathrm{As}_{2} \mathrm{~S}_{3}$-based rib waveguides. In addition, we present detailed grating growth dynamics using an in-situ monitoring setup.

Figure 1 shows the grating writing configuration. A CW, frequency-doubled, diode-pumped Nd:YAG laser at $\lambda=532 \mathrm{~nm}$ (maximum available power at the sample of $50 \mathrm{~mW}$ ) is used as light source. The (linearly polarized) beam is telescopically expanded, cylindrically focused and split using a phase mask $\left(\Lambda_{\mathrm{m}}\right.$ $=1063.3 \mathrm{~nm}$ ). The +1 and -1 orders are reflected from a pair of mirrors and interfere at the surface of the waveguide sample (TE polarized) with a spot size at the writing plane of $5.5 \times 0.6 \mathrm{~mm}^{2}$. The laser's short coherence length $(\sim 4 \mathrm{~mm})$ limits the length of the interference pattern, and results in a nearly ideal raisedapodized grating $\sim 4 \mathrm{~mm}$ in length. Tuning the Bragg wavelength of the grating can be achieved by changing the angle of the mirrors and displacement of the sample holder. Gratings are characterized with an unpolarized high-power C-band EDFA-ASE source, an auto-alignment system and a silicon detector attached to a power meter. The output of the laser source was butt-coupled into the waveguide via a highNA fiber. A second high-NA fiber coupled the transmitted output firstly to the detector for getting the best power coupling into the waveguide and then to an optical spectrum analyzer (OSA) with $60 \mathrm{pm}$ resolution bandwidth in order to measure the grating transmission spectra. A bulk optics polarization controller was placed between the sample and light source and adjusted to obtain the maximum polarization extinction ratio for the two orthogonal polarization states (TE and TM). Figure 2 shows the normalized transmission spectra of a grating written in a $4 \mu \mathrm{m}$ wide, $5 \mathrm{~cm}$ long rib waveguide ( slab height $=1.39 \mu \mathrm{m}$ and rib height $=2.39 \mu \mathrm{m}$ ) for TE polarization. The total writing power and exposure time were $6.0 \mathrm{~mW}$ and 60 seconds, respectively. From Figure 2, it is clear that this grating is strong $\left(\Delta \mathrm{n}_{\mathrm{ac}} \sim 7.7 \times 10^{-3}\right)$ and the bottom of the transmission dip is $<-33 \mathrm{~dB}$ limited by the dynamic range of our measurement setup.. The other shallow transmission dip at $\sim 1545 \mathrm{~nm}$ is due to the effect of higher order (leaky) modes supported by this waveguide.

To investigate the growth behavior of the gratings, an in-situ monitoring setup was added to the Sagnac interferometer writing system. This consists of two 3 -axis translation stages to couple the light from an EDFA-ASE source to the waveguide and from the waveguide to an OSA, via high-NA fibers. Figure 3 shows the evolution of the gratings during the writing process, for a $5 \mathrm{~cm}$ long, $5 \mu \mathrm{m}$ wide waveguide. The total writing power at the waveguide surface is $5.6 \mathrm{~mW}$. The spectra in Figure 3 are for TE polarized light, obtained with a polarization control stage (before the waveguide) in the in-situ monitoring setup. The transmission spectra of gratings are after 86, 196, 350 and 712 seconds of writing time. As seen, the Bragg wavelength, strength and shape of the spectra evolve over time. The emergence of spectral sidelobes at the short wavelength side of the spectrum is an indication of formation of a Gaussian grating profile. Change in 
Bragg wavelength is related to variation of the DC refractive index change over time $\left(\Delta \mathrm{n}_{\mathrm{dc}}(\mathrm{t})\right)$ and the width of the grating is related to amplitude of the refractive index variations $\left(\Delta \mathrm{n}_{\mathrm{ac}}(\mathrm{t})\right)$. Figure 4 shows the refractive index change (DC and AC) over time calculated based on the spectral data. As can be seen, $\Delta \mathrm{n}_{\mathrm{dc}}(\mathrm{t})$ increases in time, saturating $(\sim 0.012)$ at longer times $(\mathrm{t}>580 \mathrm{sec})$. During the grating growth, $\Delta \mathrm{n}_{\mathrm{ac}}(\mathrm{t})$ increases to a maximum value $(\sim 0.008 @ 320 \mathrm{sec})$ and then decreases for longer exposure times. This phenomenon is an indication of decreasing interference fringe visibility, due to material optical response as a result of photoexposure (photodarkrning) [7].

In conclusion, we present very strong, well apodized gratings written in chalcogenide glass rib waveguides, appropriate for advanced signal processing applications, with a modified Sagnac interferometer. In addition, we present detailed measurements for grating evolution during the writing process.

\section{References:}

[1] A. Zakery and S.R. Elliott, J. Non-Crystal. Solids, 330 (2003) 1-12.

[2] M. Asobe, Opt. Fiber Technol., 3 (1997) 142-148.

[3] A. Saliminia, K. Le Foulgoc, A. Villeneuve and T. Galstian, Fiber Integrat. Opt., 20 (2001) 151-158.

[4] A. Zakery, J. Phys. D: Appl. Phys., 35 (2002) 2909-2913.

[5] M. Asobe, T. Ohara, I. Yokohama and T. Kaino, Electron. Lett., 32 (1996) 1611-1613.

[6] N. Ponnampalam, R. G. DeCorby, H. T. Nguyen, P. K. Dwivedi, C. J. Haugen, J. N. McMullin, and S. O. Kasap, Opt. Express, 12 (2004) 6270-6277.

[7] T. G. Robinson, R. G. DeCorby, J. N. McMullin, C. J. Haugen, S. O. Kasap and D. Tonchev, Opt. Lett., 28 (2003) 459-461.

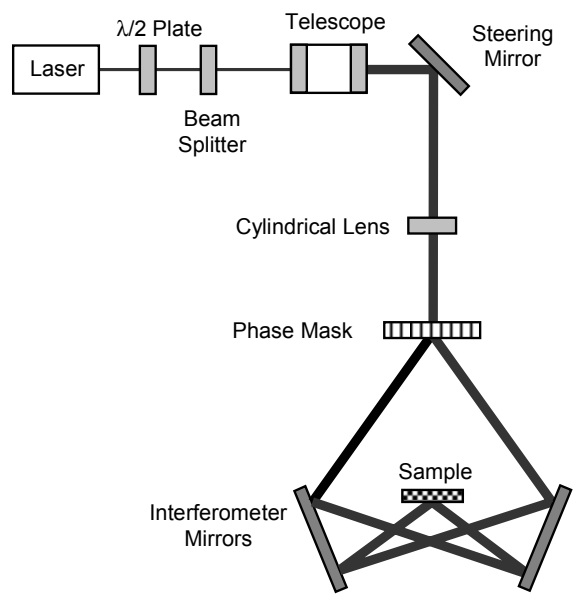

Figure 1. Grating Writing setup based on the modified Sagnac interferometer.

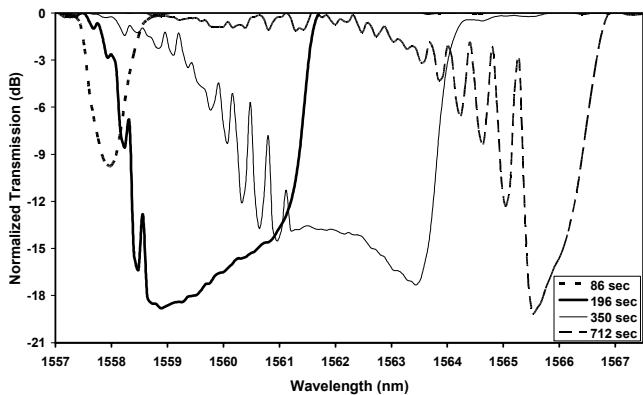

Figure 3. Normalized transmission spectra of the grating (TE polarization) monitored by in-situ monitoring setup at $86,196,350$ and $712 \mathrm{sec}$.

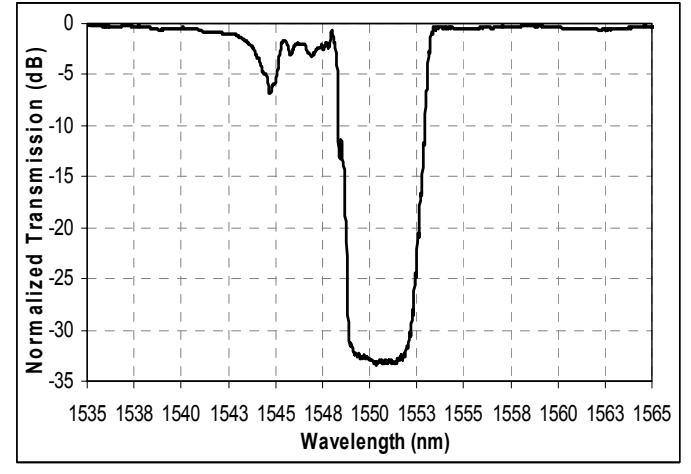

Figure 2. Normalized transmission spectra of the grating (TE polarization) written in $\mathrm{As}_{2} \mathrm{~S}_{3}$-based rib waveguide.

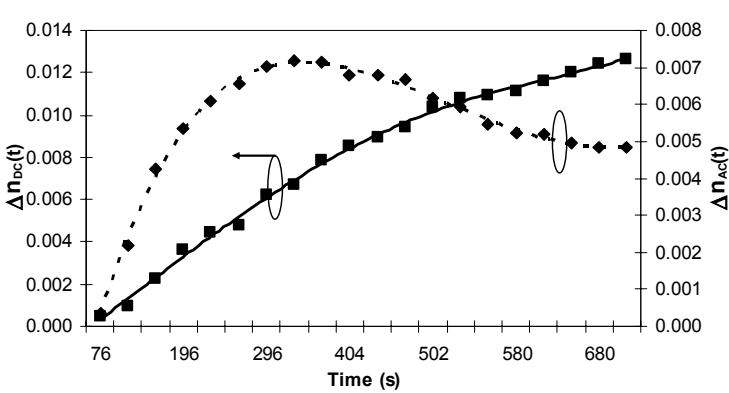

Figure 4. Variation of $\Delta \mathrm{n}_{\mathrm{dc}}$ and $\Delta \mathrm{n}_{\mathrm{ac}}$ versus exposure time. 